\title{
Approximate solutions for nonconvex set-valued optimization and vector variational inequality
}

\section{Guolin Yu* and Xiangyu Kong}

*Correspondence:

guolin_yu@126.com

Institute of Applied Mathematics,

Beifang University of Nationalities,

Yinchuan, Ningxia 750021, P.R. China

\begin{abstract}
This note deals with approximate solutions in vector optimization involving a generalized cone-invex set-valued mapping. First, a new class of generalized cone-invex set-valued maps, called cone-subinvex set-valued maps, is introduced. Then the sufficient optimality condition and two types dual theorems are established for weakly approximate minimizers under the assumption of cone-subinvexity. Finally, it also reveals the closed relationships between a weakly approximate minimizer of a cone-subinvex set-valued optimization problem and a weakly approximate solution of a kind of vector variational inequality.
\end{abstract}

MSC: Primary 90C29; 90C46; secondary 26B25

Keywords: generalized invex set-valued mappings; optimality conditions; contingent epiderivative; approximate solutions

\section{Introduction}

Recently, there has been an increasing interest in the extension of vector optimization to set-valued optimization. As a bridge between different areas of optimization, the theory of set-valued optimization problems has wide applications in differential inclusion, variational inequality, optimal control, game theory, economic equilibrium problem, decision making, etc. For more details of set-valued optimization theory and applications, the reader can refer to the excellent books [1-4].

The derivative of set-valued maps is most important for the formulation of optimality conditions. Aubin and Frankowsa [1] introduced the notion of a contingent derivative of a set-valued map as an extension of the concept of Fréchet differentiability. From then on, various approaches have been followed in defining the concept of derivative for set-valued maps. Among these notions, a meaningful and useful concept is the contingent epiderivative, which was given by Jahn and Rauh [5]. It is important to note that the contingent epiderivative is a single-valued map. Recently, much attention has been paid to characterizing optimality conditions for set-valued optimization and related problems by utilizing contingent epiderivatives; for example, see [6-12]. On the other hand, convex analysis is a powerful tool for the investigation of optimal solutions of vector optimization problems. Various notions of generalized convexity have been introduced to weaken convexity. One

(c) 2015 Yu and Kong. This article is distributed under the terms of the Creative Commons Attribution 4.0 International License (http://creativecommons.org/licenses/by/4.0/), which permits unrestricted use, distribution, and reproduction in any medium, provided you give appropriate credit to the original author(s) and the source, provide a link to the Creative Commons license, and indicate if changes were made. 
of such generalizations is invexity, which was firstly introduced by Hanson [13] for nonlinear programming. Based upon this concept, some scholars developed further generalizations of invexity to vector optimization involving set-valued maps. For example, Luc and Malivert [14] extended the concept of invexity to set-valued optimization and investigated the necessary and sufficient optimality conditions; Sach and Craven [15] proved duality theorems for set-valued optimization problems under invexity assumptions. In [16-19], optimality conditions and a characterization of solution sets of set-valued optimization problems involving generalized invexity are investigated.

Since duality assertions allow us to study a minimization problem through a maximization problem and to know what one can expect in the best case. At the same time, duality has resulted in many applications within optimization, and it has provided many unifying conceptual insights into economics and management science. So, it is not surprising that duality is one of the important topics in set-valued optimization. There are many papers dedicated to duality theory of set-valued optimization, for instance, [20-25] cited in this paper are closely related to the present work.

On the other hand, since it has been introduced by Giannessi [26], the theory of vector variational inequalities has shown many applications in vector optimization problems and traffic equilibrium problems. In fact, some recent work has shown that optimality conditions of some vector optimization problems can be characterized by vector variational inequalities. For example, Al-Homidan and Ansari [27] dealt with different kinds of generalized vector variational-like inequality problems and a vector optimization problem. Some relationships between the solutions of generalized vector variational-like inequality problem and an efficient solution of a vector optimization problem have been established; Ansari et al. [28] worked on the generalized vector variational-like inequalities involving the Dini subdifferential, and some relations among these inequalities and vector optimization problems are presented. In the literature $[29,30]$, the authors focused on the exponential type vector variational-like inequalities and vector optimization problems with exponential type invexities. Observing the above mentioned papers, we found that the invexity plays exactly the same role in variational-like inequalities as the classical convexity plays in variational inequalities. Motivated by this work, this paper will extend the partial results to the setting of a set-valued mapping under the weaker invexity assumption.

In addition, approximate solutions of optimization problems are very important from both the theoretical and the practical points of view because they exist under very mild hypotheses and a lot of solution methods propose this kind of solutions. Thus, it is meaningful to consider various concepts of approximate solutions to set-valued optimization problems. Recently, approximate solutions for set-valued optimization have caught many scholars' attention; for example, see [31-34] and the references therein.

Based upon the above observation, the purpose of this paper is two aspects: first, to introduce a new class of generalized set-valued cone-invex maps and establish sufficient optimal conditions and dual theorems of approximate solutions for set-valued optimization problems under these generalized convexities; second, to study the optimality conditions of weakly approximate minimizer in vector optimization involving generalized cone-invex set-valued mappings by using the notions of vector variational inequality.

This paper is structured as follows: In Section 2, some well-known definitions and results used in the sequel are recalled; a new class of generalized set-valued cone-invex maps, 
named cone-subinvex set-valued maps, is introduced. In Section 3 and Section 4, we give the sufficient optimality conditions and two types dual theorems of weakly approximate minimizers, respectively. Section 5 is devoted to revealing the closed relation between weakly approximate solutions of vector optimization and variational inequality involving set-valued cone-subinvex mappings.

\section{Preliminaries}

In this paper, $X, Y$, and $Z$ are assumed to be Banach spaces with topological dual $X^{*}, Y^{*}$, and $Z^{*}$, respectively. For any $x \in X$ and $x^{*} \in X^{*}$, the canonical form between $X$ and $X^{*}$ is denoted by $x^{* T} x$. Let $D \subset Y$ and $E \subset Z$ be pointed closed convex cones with $\operatorname{int}(D) \neq \emptyset$. We write

$$
D^{*}=\left\{y^{*}: y^{* T} d \geq 0, \forall d \in D\right\}
$$

and similarly for $E^{*}$. Let $F: X \rightarrow 2^{Y}$ be a set-valued mapping. The set

$$
\operatorname{dom}(F):=\{x \in X: F(x) \neq \emptyset\}
$$

is called the domain of $F$. The set

$$
\operatorname{graph}(F):=\{(x, y) \in X \times Y: y \in F(x)\}
$$

is called the graph of the map $F$. The set

$$
\operatorname{epi}(F):=\{(x, y) \in X \times Y: y \in F(x)+D\}
$$

is called the epigraph of $F$.

Let $(\bar{x}, \bar{y}) \in \operatorname{graph}(F)$. The contingent cone (see [5]) to epi $(F)$ at $(\bar{x}, \bar{y})$ denoted by $T(\operatorname{epi}(F),(\bar{x}, \bar{y}))$, which consists of all tangent vectors

$$
(x, y):=\lim _{n \rightarrow \infty} \lambda_{n}\left(\left(x_{n}, y_{n}\right)-(\bar{x}, \bar{y})\right)
$$

where $(\bar{x}, \bar{y})=\lim _{n \rightarrow \infty}\left(x_{n}, y_{n}\right),\left(x_{n}, y_{n}\right) \in \operatorname{epi}(F), \lambda_{n}>0$, for all $n \in \mathbb{N}$.

Definition 2.1 (see [5]) Let a pair $(\bar{x}, \bar{y}) \in \operatorname{graph}(F)$ be given. A single-valued map $D F(\bar{x}, \bar{y})$ : $X \rightarrow Y$ whose epigraph equals the contingent cone to the epigraph of $F$ at $(\bar{x}, \bar{y})$, that is,

$$
\operatorname{epi}(D F(\bar{x}, \bar{y}))=T(\operatorname{epi}(F),(\bar{x}, \bar{y}))
$$

is called contingent epiderivative of $F$ at $(\bar{x}, \bar{y})$. If the contingent epiderivative of $F$ at any point in graph $(F)$ exists, then we say $F$ is a contingent epiderivable set-valued map.

Next, we begin with recalling the concepts of a convex set and an invex set. It is well known that a subset $S$ of $X$ is a convex set, if for any $x, z \in S, t \in[0,1]$, we have $t z+(1-t) x \in S$. 
Definition 2.2 (see [14]) A subset $S \subset X$ is said to be an $\eta$-invex set, if, for every $x \in S$, $z \in S$, there exists a map $\eta: X \times X \rightarrow X$ such that $z+t \eta(x, z) \in S$, for all $t \in[0,1]$.

Example 2.3 Let $S=(0,+\infty) \subset \mathbb{R}=X$. Then $S$ is invex with respect to $\eta: X \times X \rightarrow X$, $\eta(x, y)=x+y$.

In Definition 2.2, when $\eta(x, z)=x-z$, then $S$ is a convex set. In general, the opposite is not true.

Example 2.4 Suppose that $S=[-5,-2] \cup[2,10]$, then $S$ is an invex with respect to $\eta(x, y)$, defined by

$$
\eta(x, y)= \begin{cases}x-y, & \text { if } x \leq 0, y \leq 0 \\ x-y, & \text { if } x \geq 0, y \geq 0 \\ -5-y, & \text { if } x \geq 0, y \leq 0 \\ 2-y, & \text { if } x \leq 0, y \geq 0\end{cases}
$$

Obviously, $S$ is not a convex set.

Definition 2.5 (see [14]) Let $\eta: X \times X \rightarrow X$ be a map and $F: X \rightarrow 2^{Y}$ be a contingent epiderivable set-valued map at a point $(\bar{x}, \bar{y}) \in \operatorname{graph}(F)$ with $\operatorname{dom}(D F(\bar{x}, \bar{y}))=X$. Then $F$ is said to be a $D$ - $\eta$-invex map at $(\bar{x}, \bar{y})$ if

$$
F(x)-\bar{y} \subset D F(\bar{x}, \bar{y})(\eta(x, \bar{x}))+D, \quad \text { for all } x \in X .
$$

$F$ is said to be a $D-\eta$-invex map at $\bar{x}$, if (2.1) holds for any $\bar{y} \in F(\bar{x})$.

Example 2.6 Let $X=\mathbb{R}_{+}, Y=\mathbb{R}^{2}, D=\mathbb{R}_{+}^{2}$, and $F: \mathbb{R}_{+} \rightarrow 2^{\mathbb{R}^{2}}$ be defined by

$$
F(x)=\left\{\left(x^{2}, x\right), x \geq 0\right\}
$$

and the epigraph of $F$

$$
\operatorname{epi}(F)=\left\{\left(x,\left(y_{1}, y_{2}\right)\right) \in \mathbb{R}_{+} \times \mathbb{R}_{+}^{2}:\left(y_{1}, y_{2}\right) \in F(x)+\mathbb{R}_{+}^{2}\right\}
$$

Let $0=\bar{x} \in \mathbb{R}_{+}$and $(0,0)=\bar{y}=\left(\bar{y}_{1}, \overline{y_{2}}\right) \in F(\bar{x})$. By calculating, we have

$$
T(\operatorname{epi}(F),(0,(0,0)))=\mathbb{R}_{+} \times \mathbb{R}_{+}^{2} .
$$

Hence, for any $x \in \mathbb{R}_{+}$,

$$
D F(0,(0,0))(x)=\{(0,0)\}
$$

Therefore, $D F(0,(0,0))$ exists for any $x \in \mathbb{R}_{+}$and for any map $\eta: \mathbb{R}_{+} \times \mathbb{R}_{+} \rightarrow \mathbb{R}_{+}$, one has

$$
\left(x^{2}, x\right)-(0,0) \in\{(0,0)\}+\mathbb{R}_{+}^{2}, \quad \text { for all } y \geq 0 .
$$


Hence,

$$
F(x)-(0,0) \subset D F(0,(0,0))(\eta(x, 0))+\mathbb{R}_{+}^{2}, \quad \text { for all } x \in \mathbb{R}_{+} .
$$

So, $F$ is a $D$ - $\eta$-invex map at $(\bar{x}, \bar{y})=(0,(0,0))$ with respect to any mapping $\eta$.

Definition 2.7 Suppose that $\eta: X \times X \rightarrow X, e \in \operatorname{int}(D), \varepsilon \geq 0$, and $F: X \rightarrow 2^{Y}$ is a contingent epiderivable set-valued map at a point $(\bar{x}, \bar{y}) \in \operatorname{graph}(F)$ with $\operatorname{dom}(D F(\bar{x}, \bar{y}))=X$. Then $F$ is said to be a $D-\eta$-subinvex set-valued mapping at $(\bar{x}, \bar{y})$ with respect to $\varepsilon \cdot e$, if

$$
\varepsilon \cdot e+F(x)-\bar{y} \subset D F(\bar{x}, \bar{y})(\eta(x, \bar{x}))+D, \quad \text { for all } x \in X \text {. }
$$

$F$ is said to be a $D-\eta$-subinvex map at $\bar{x}$ with respect to $\varepsilon \cdot e$, if $(2.2)$ holds for any $\bar{y} \in F(\bar{x})$.

Obviously, if $F$ is a $D$ - $\eta$-invex map at $(\bar{x}, \bar{y}) \in \operatorname{graph}(F)$, then $F$ is a $D$ - $\eta$-subinvex setvalued mapping at $(\bar{x}, \bar{y})$ with respect to $\varepsilon \cdot e$. However, the inverse proposition is not necessarily true, as is illustrated in the following example.

Example 2.8 Let $X=\mathbb{R}, Y=\mathbb{R}^{2}, D=\mathbb{R}_{+}^{2}$, and $F: \mathbb{R} \rightarrow 2^{\mathbb{R}^{2}}$ be defined by

$$
F(x)= \begin{cases}\{(y, \sqrt{y}): y \geq 0\}, & \text { if } x \geq 0 \\ \{(y, \sqrt{-y}): y \in[-4,0]\}, & \text { if } x<0\end{cases}
$$

and the epigraph of $F$

$$
\operatorname{epi}(F)=\left\{\left(x,\left(y_{1}, y_{2}\right)\right) \in \mathbb{R} \times \mathbb{R}^{2}:\left(y_{1}, y_{2}\right) \in F(x)+\mathbb{R}_{+}^{2}\right\}
$$

Let $0=\bar{x} \in \mathbb{R}$ and $(0,0)=\bar{y}=\left(\overline{y_{1}}, \overline{y_{2}}\right) \in F(\bar{x})$, we get

$$
T(\operatorname{epi}(F),(0,(0,0)))=\mathbb{R} \times \mathbb{R}_{+}^{2}
$$

and

$$
D F(0,(0,0))(x)=\{(0,0)\}, \quad \forall x \in \mathbb{R} .
$$

Thus, $D F(0,(0,0))$ exists for any $x \in \mathbb{R}$. However, for any map $\eta: \mathbb{R} \times \mathbb{R} \rightarrow \mathbb{R}$, we have

$$
(y,|\sqrt{-y}|)-(0,0) \notin\{(0,0)\}+\mathbb{R}_{+}^{2}, \quad \text { for all } y \in[-4,0] .
$$

Hence,

$$
F(x)-(0,0) \not \subset D F(0,(0,0))(\eta(x, 0))+\mathbb{R}_{+}^{2}, \quad \text { for all } x \in \mathbb{R}
$$

which indicates that $F$ is not $D-\eta$-invex at $(\bar{x}, \bar{y})=(0,(0,0))$ with respect to any mapping $\eta$.

Now, choosing any $\eta, e=(1,1) \in \operatorname{int}\left(\mathbb{R}_{+}^{2}\right)$, and $\varepsilon \geq 4$ (or, $e=(4,4) \in \operatorname{int}\left(\mathbb{R}_{+}^{2}\right)$ and $\varepsilon \geq 1$ ), we have

$$
\varepsilon \cdot e+(y,|\sqrt{y}|)-(0,0) \in\{(0,0)\}+\mathbb{R}_{+}^{2}, \quad \text { for all } y \geq 0, x \geq 0,
$$


and

$$
\varepsilon \cdot e+(y,|\sqrt{-y}|)-(0,0) \in\{(0,0)\}+\mathbb{R}_{+}^{2}, \quad \text { for all } y \in[-4,0], x<0
$$

So, we get

$$
\varepsilon \cdot e+F(x)-(0,0) \subset D F(0,(0,0))(\eta(x, 0))+\mathbb{R}_{+}^{2}, \quad \text { for all } x \in \mathbb{R}
$$

Hence, $F$ is a $D-\eta$-subinvex set-valued mapping at $(0,(0,0))$ with respect to $\varepsilon \cdot e$.

\section{Sufficient optimality conditions}

Let $F: X \rightarrow 2^{Y}$ and $G: X \rightarrow 2^{Z}$ be two set-valued maps with $\operatorname{dom}(F)=\operatorname{dom}(G)=X$. In Section 3 and Section 4 , we consider the following set-valued optimization problem:

$$
\text { (SOP-I) } \begin{cases}\text { minimize } & F(x) \\ \text { subject to } & G(x) \cap(-E) \neq \emptyset, \\ & x \in X .\end{cases}
$$

A point $(\bar{x}, \bar{y}) \in X \times Y$ is said to be a feasible point of the problem (SOP-I) if $\bar{x} \in X, \bar{y} \in$ $F(\bar{x})$, and $G(\bar{x}) \cap(-E) \neq \emptyset$. Let $\Omega=\{x \in X:(x, y)$ is a feasible point of the problem (SOP-I) . Then the weakly approximate minimizer for the set-valued optimization problem (SOP-I) is defined in the following way.

Definition 3.1 (see [21]) (i) A point $\bar{x} \in \Omega$ is said to be a weak efficient solution of the problem (SOP-I), if there exists $\bar{y} \in F(\bar{x})$ such that

$$
(F(\Omega)-\bar{y}) \cap(-\operatorname{int}(D))=\emptyset,
$$

and the pair $(\bar{x}, \bar{y}) \in \operatorname{graph}(F)$ is said to be a weak minimizer of (SOP-I).

(ii) Let $\varepsilon \geq 0$ and $e \in \operatorname{int}(D)$. A point $\bar{x} \in \Omega$ is said to be a weak $\varepsilon \cdot e$-efficient solution of the problem (SOP-I), if there exists $\bar{y} \in F(\bar{x})$ such that

$$
(F(\Omega)-\bar{y}+\varepsilon \cdot e) \cap(-\operatorname{int}(D))=\emptyset,
$$

and the pair $(\bar{x}, \bar{y}) \in \operatorname{graph}(F)$ is said to be a weak $\varepsilon \cdot e$-minimizer of (SOP-I).

Theorem 3.2 (Sufficient optimality condition) Let $e \in \operatorname{int}(D), \varepsilon \geq 0$, and $(\bar{x}, \bar{y}) \in \operatorname{graph}(F)$ be a feasible point of the problem (SOP-I) and $\bar{z} \in G(\bar{x}) \cap(-E)$. Assume that the contingent epiderivatives $D F(\bar{x}, \bar{y})$ and $D G(\bar{x}, \bar{z})$ exist with $\operatorname{dom}(D F(\bar{x}, \bar{y}))=\operatorname{dom}(D G(\bar{x}, \bar{z}))=X$. Let $F$ be D- $\eta$-subinvex at $(\bar{x}, \bar{y})$ with respect to $\varepsilon \cdot e$ and $G$ be $E$ - $\eta$-invex at $(\bar{x}, \bar{z})$ with respect to the same $\eta$. If there exists $\left(y^{*}, z^{*}\right) \in D^{*} \times E^{*}$ with $y^{*} \neq 0_{Y^{*}}$ such that

$$
y^{* T} D F(\bar{x}, \bar{y})(\eta(x, \bar{x}))+z^{* T} D G(\bar{x}, \bar{z})(\eta(x, \bar{x})) \geq 0, \quad \forall x \in X,
$$

and

$$
z^{* T} \bar{z}=0
$$

then $(\bar{x}, \bar{y})$ is a weak $\varepsilon \cdot e$-minimizer of the problem (SOP-I). 
Proof Assuming that $(\bar{x}, \bar{y})$ is not a weak $\varepsilon \cdot e$-minimizer of the problem (SOP-I),

$$
(F(\Omega)-\bar{y}+\varepsilon \cdot e) \cap(-\operatorname{int}(D)) \neq \emptyset .
$$

Hence, there exist $\hat{x} \in \Omega$ and $\hat{y} \in F(\hat{x})$ such that

$$
\hat{y}-\bar{y}+\varepsilon \cdot e \in-\operatorname{int}(D)
$$

Noticing that $y^{*} \in D^{*} \backslash\left\{0_{Y^{*}}\right\}$, we get

$$
y^{* T}(\hat{y}-\bar{y}+\varepsilon \cdot e)<0 .
$$

On the other hand, since $F$ is $D-\eta$-subinvex at $(\bar{x}, \bar{y})$ with respect to $\varepsilon \cdot e$, we obtain

$$
F(\hat{x})-\bar{y}+\varepsilon \cdot e \subset D F(\bar{x}, \bar{y})(\eta(\hat{x}, \bar{x}))+D .
$$

Therefore,

$$
\hat{y}-\bar{y}+\varepsilon \cdot e \in D F(\bar{x}, \bar{y})(\eta(\hat{x}, \bar{x}))+D .
$$

So, we get

$$
0>y^{* T}(\hat{y}-\bar{y}+\varepsilon \cdot e) \geq y^{* T} D F(\bar{x}, \bar{y})(\eta(\hat{x}, \bar{x})),
$$

and then

$$
y^{* T} D F(\bar{x}, \bar{y})(\eta(\hat{x}, \bar{x}))<0 .
$$

Again, since $G$ is $E-\eta$-invex at $(\bar{x}, \bar{z})$, we have

$$
G(\hat{x})-\bar{z} \subset D G(\bar{x}, \bar{z})(\eta(\hat{x}, \bar{x}))+E .
$$

Because $\hat{x} \in \Omega$, there exists an element $\hat{z} \in G(\hat{x}) \cap(-E)$ such that

$$
\hat{z}-\bar{z} \in D G(\bar{x}, \bar{z})(\eta(\hat{x}, \bar{x}))+E .
$$

It follows from $z^{*} \in E^{*}$ that

$$
z^{* T}(\hat{z}-\bar{z}) \geq z^{* T} D G(\bar{x}, \bar{z})(\eta(\hat{x}, \bar{x})) .
$$

Since $z^{* T} \hat{z} \leq 0$ and $z^{* T} \bar{z}=0$, we get $z^{* T}(\hat{z}-\bar{z}) \leq 0$ and

$$
z^{* T} D G(\bar{x}, \bar{z})(\eta(\hat{x}, \bar{x})) \leq 0 .
$$

So, we have from (3.3) and (3.4)

$$
y^{* T} D F(\bar{x}, \bar{y})(\eta(\hat{x}, \bar{x}))+z^{* T} D G(\bar{x}, \bar{z})(\eta(\hat{x}, \bar{x}))<0,
$$

which contradicts (3.1). Hence, $(\bar{x}, \bar{y})$ is a weak $\varepsilon \cdot e$-minimizer of the problem (SOP-I). 


\section{Duality theorems}

\subsection{Mond-Weir type duality}

For the primal problem (SOP-I), this subsection considers the Mond-Weir dual problem (MWD):

(MWD) $\begin{cases}\text { maximize } & y^{\prime} \\ \text { subject to } & y^{* T} D F\left(x^{\prime}, y^{\prime}\right)\left(\eta\left(x, x^{\prime}\right)\right)+z^{* T} D G\left(x^{\prime}, z^{\prime}\right)\left(\eta\left(x, x^{\prime}\right)\right) \geq 0, \quad \forall x \in X, \\ & z^{* T} z^{\prime} \geq 0,\left(y^{*}, z^{*}\right) \in\left(D^{*} \backslash\left\{0_{Y^{*}}\right\}\right) \times E^{*}, \\ & x^{\prime} \in X, y^{\prime} \in F\left(x^{\prime}\right) \text { and } z^{\prime} \in G\left(x^{\prime}\right) .\end{cases}$

A point $\left(x^{\prime}, y^{\prime}, z^{\prime}, y^{*}, z^{*}\right)$ satisfying all the constraints of the problem (MWD) is called a feasible point of the problem (MWD). Let $K_{1}=\left\{y^{\prime}:\left(x^{\prime}, y^{\prime}, z^{\prime}, y^{*}, z^{*}\right)\right.$ is a feasible point of (MWD)\}.

Definition 4.1 Let $\varepsilon \geq 0$ and $e \in \operatorname{int}(D)$. A feasible point $\left(x^{\prime}, y^{\prime}, z^{\prime}, y^{*}, z^{*}\right)$ of the problem (MWD) is called a weak $\varepsilon \cdot e$-maximizer of (MWD) if

$$
\left(K_{1}-y^{\prime}-\varepsilon \cdot e\right) \cap \operatorname{int}(D)=\emptyset .
$$

Theorem 4.2 (Weak duality) Let $e \in \operatorname{int}(D), \varepsilon \geq 0,(\bar{x}, \bar{y})$, and $\left(x^{\prime}, y^{\prime}, z^{\prime}, y^{*}, z^{*}\right)$ be feasible points for (SOP-I) and (MWD), respectively. Suppose that $F$ is D- $\eta$-subinvex at $\left(x^{\prime}, y^{\prime}\right)$ with respect to $\varepsilon \cdot e$ and $G$ is $E$ - $\eta$-invex at $\left(x^{\prime}, z^{\prime}\right)$ with respect to the same $\eta$. Then we have

$$
\bar{y}-y^{\prime}+\varepsilon \cdot e \notin-\operatorname{int}(D)
$$

Proof We proceed by contradiction. Suppose that

$$
\bar{y}-y^{\prime}+\varepsilon \cdot e \in-\operatorname{int}(D)
$$

Since $y^{*} \in D^{*} \backslash\left\{0_{Y^{*}}\right\}$, we have

$$
y^{* T}\left(\bar{y}-y^{\prime}+\varepsilon \cdot e\right)<0 .
$$

And because $\bar{x}$ is feasible point for (SOP-I), we get $G(\bar{x}) \cap(-E) \neq \emptyset$. Hence, there exists $\bar{z} \in G(\bar{x}) \cap(-E)$ such that $z^{* T} \bar{z} \leq 0$. On the other hand, we have from the dual constraint of (MWD) $-z^{* T} z^{\prime} \leq 0$. Therefore,

$$
z^{* T}\left(\bar{z}-z^{\prime}\right) \leq 0
$$

We get from (4.2) and (4.3)

$$
y^{* T}\left(\bar{y}-y^{\prime}+\varepsilon e\right)+z^{* T}\left(\bar{z}-z^{\prime}\right)<0 .
$$

Now, since $F$ is $D$ - $\eta$-subinvex at $\left(x^{\prime}, y^{\prime}\right)$ with respect to $\varepsilon \cdot e$ and $G$ is $E$ - $\eta$-invex at $\left(x^{\prime}, z^{\prime}\right)$, we derive

$$
F(\bar{x})-y^{\prime}+\varepsilon \cdot e \subset D F\left(x^{\prime}, y^{\prime}\right)\left(\eta\left(\bar{x}, x^{\prime}\right)\right)+D
$$


and

$$
G(\bar{x})-z^{\prime} \subset D G\left(x^{\prime}, z^{\prime}\right)\left(\eta\left(\bar{x}, x^{\prime}\right)\right)+E .
$$

Therefore, we obtain

$$
y^{* T}\left(\bar{y}-y^{\prime}+\varepsilon \cdot e\right) \geq y^{* T} D F\left(x^{\prime}, y^{\prime}\right)\left(\eta\left(\bar{x}, x^{\prime}\right)\right),
$$

and

$$
z^{* T}\left(\bar{z}-z^{\prime}\right) \geq z^{* T} D G\left(x^{\prime}, z^{\prime}\right)\left(\eta\left(\bar{x}, x^{\prime}\right)\right) .
$$

Furthermore, we find from (4.4), (4.5), and (4.6) that

$$
y^{* T} D F\left(x^{\prime}, y^{\prime}\right)\left(\eta\left(\bar{x}, x^{\prime}\right)\right)+z^{* T} D G\left(x^{\prime}, z^{\prime}\right)\left(\eta\left(\bar{x}, x^{\prime}\right)\right)<0,
$$

which contradicts the dual constraint of (MWD). So, (4.1) is satisfied and this completes the proof.

Theorem 4.3 (Strong duality) Let $e \in \operatorname{int}(D), \varepsilon \geq 0$, and $(\bar{x}, \bar{y})$ be a weak $\varepsilon \cdot$ e-minimizer of (SOP-I). Suppose that, for some $\left(y^{*}, z^{*}\right) \in\left(D^{*} \backslash\left\{0_{Y^{*}}\right\}\right) \times E^{*}$ and $\bar{z} \in G(\bar{x}) \cap(-E)$, (3.1) and (3.2) are satisfied. Then $\left(\bar{x}, \bar{y}, \bar{z}, y^{*}, z^{*}\right)$ is a feasible point for (MWD). Furthermore, if the weak duality theorem, Theorem 4.2, between (SOP-I) and (MWD) holds, then $\left(\bar{x}, \bar{y}, \bar{z}, y^{*}, z^{*}\right.$ ) is a weak $\varepsilon \cdot e$-maximizer of (MWD).

Proof Since (3.1) and (3.2) hold, it is obvious that $\left(\bar{x}, \bar{y}, \bar{z}, y^{*}, z^{*}\right)$ is a feasible point for (MWD). Afterwards, we will prove that

$$
\left(K_{1}-\bar{y}-\varepsilon \cdot e\right) \cap \operatorname{int}(D)=\emptyset
$$

In fact, assume that there exists $y^{\prime} \in K_{1}$ such that

$$
y^{\prime}-\bar{y}-\varepsilon \cdot e \in \operatorname{int}(D)
$$

This contradicts the weak duality theorem, Theorem 4.2, between (SOP-I) and (MWD).

Theorem 4.4 (Converse duality) Let $e \in \operatorname{int}(D), \varepsilon \geq 0$, and $\left(x^{\prime}, y^{\prime}, z^{\prime}, y^{*}, z^{*}\right)$ be a feasible point of the problem (MWD) and $z^{\prime} \in G\left(x^{\prime}\right) \cap(-E)$. Suppose that $F$ is D- $\eta$-subinvex at $\left(x^{\prime}, y^{\prime}\right)$ with respect to $\varepsilon \cdot e$ and $G$ is $E$ - $\eta$-invex at $\left(x^{\prime}, z^{\prime}\right)$ with respect to the same $\eta$. Then $\left(x^{\prime}, y^{\prime}\right)$ is a weak $\varepsilon \cdot e$-minimizer of the problem (SOP-I).

Proof Firstly, it is clearly that $\left(x^{\prime}, y^{\prime}\right)$ is a feasible point of the problem (SOP-I). Next, assuming that $\left(x^{\prime}, y^{\prime}\right)$ is not a weak $\varepsilon \cdot e$-minimizer of the problem (SOP-I),

$$
\left(F(\Omega)-y^{\prime}+\varepsilon \cdot e\right) \cap(-\operatorname{int}(D)) \neq \emptyset .
$$


Hence, there are $\hat{x} \in \Omega$ and $\hat{y} \in F(\hat{x})$ such that

$$
\left(\hat{y}-y^{\prime}+\varepsilon \cdot e\right) \in-\operatorname{int}(D)
$$

Noticing that $y^{*} \in D^{*} \backslash\left\{0_{Y^{*}}\right\}$, we have

$$
y^{* T}\left(\hat{y}-y^{\prime}+\varepsilon \cdot e\right)<0
$$

Again, because $\hat{x} \in \Omega$, we get $G(\hat{x}) \cap(-E) \neq \emptyset$. Taking $\hat{z} \in G(\hat{x}) \cap(-E)$, we derive from $z^{*} \in E^{*}$ that $z^{* T} \hat{z} \leq 0$. By the constraint of (MWD), we have $z^{* T} z^{\prime} \geq 0$. Therefore, we get

$$
z^{* T}\left(\hat{z}-z^{\prime}\right) \leq 0
$$

Together with (4.7), we obtain

$$
y^{* T}\left(\hat{y}-y^{\prime}+\varepsilon \cdot e\right)+z^{* T}\left(\hat{z}-z^{\prime}\right)<0 .
$$

On the other hand, since $F$ is $D$ - $\eta$-subinvex at $\left(x^{\prime}, y^{\prime}\right)$ with respect to $\varepsilon \cdot e$ and $G$ is $E$ - $\eta$-invex at $\left(x^{\prime}, z^{\prime}\right)$, it follows that

$$
F(\hat{x})-y^{\prime}+\varepsilon \cdot e \subset D F\left(x^{\prime}, y^{\prime}\right)\left(\eta\left(\hat{x}, x^{\prime}\right)\right)+D
$$

and

$$
G(\hat{x})-z^{\prime} \subset D G\left(x^{\prime}, z^{\prime}\right)\left(\eta\left(\hat{x}, x^{\prime}\right)\right)+E .
$$

Thus, combining with (4.8), we get

$$
0>y^{* T}\left(\hat{y}-y^{\prime}+\varepsilon \cdot e\right)+z^{* T}\left(\hat{z}-z^{\prime}\right) \geq y^{* T} D F\left(x^{\prime}, y^{\prime}\right)\left(\eta\left(\hat{x}, x^{\prime}\right)\right)+z^{* T} D G\left(x^{\prime}, z^{\prime}\right)\left(\eta\left(\hat{x}, x^{\prime}\right)\right) .
$$

So,

$$
y^{* T} D F\left(x^{\prime}, y^{\prime}\right)\left(\eta\left(\hat{x}, x^{\prime}\right)\right)+z^{* T} D G\left(x^{\prime}, z^{\prime}\right)\left(\eta\left(\hat{x}, x^{\prime}\right)\right)<0,
$$

which contradicts the dual constraint of (MWD). Hence, $\left(x^{\prime}, y^{\prime}\right)$ is a weak $\varepsilon \cdot e$-minimizer of the problem (SOP-I).

\subsection{Wolfe type duality}

Let us fix a point $d_{0} \in D \backslash\left\{0_{Y}\right\}$ and consider the following problem (WD), called the Wolfe type dual problem of (SOP-I):

$$
\text { (WD) } \begin{cases}\text { maximize } & y^{\prime}+z^{* T} z^{\prime} \cdot d_{0} \\ \text { subject to } & y^{* T} D F\left(x^{\prime}, y^{\prime}\right)\left(\eta\left(x, x^{\prime}\right)\right)+z^{* T} D G\left(x^{\prime}, z^{\prime}\right)\left(\eta\left(x, x^{\prime}\right)\right) \geq 0, \quad \forall x \in X, \\ & y^{* T} d_{0}=1\left(y^{*}, z^{*}\right) \in\left(D^{*} \backslash\left\{0_{Y^{*}}\right\}\right) \times E^{*}, \\ & x^{\prime} \in X, y^{\prime} \in F\left(x^{\prime}\right) \text { and } z^{\prime} \in G\left(x^{\prime}\right) .\end{cases}
$$


A point $\left(x^{\prime}, y^{\prime}, z^{\prime}, y^{*}, z^{*}\right)$ satisfying all the constraints of problem (WD) is called a feasible point of the problem (WD). Let $K_{2}=\left\{y^{\prime}+z^{* T} z^{\prime} \cdot d_{0}:\left(x^{\prime}, y^{\prime}, z^{\prime}, y^{*}, z^{*}\right)\right.$ is a feasible point of (WD)\}.

Definition 4.5 Let $\varepsilon \geq 0$ and $e \in \operatorname{int}(D)$. A feasible point $\left(x^{\prime}, y^{\prime}, z^{\prime}, y^{*}, z^{*}\right)$ of the problem (WD) is called to be a weak $\varepsilon \cdot e$-maximizer of (WD) if

$$
\left(K_{2}-\left(y^{\prime}+z^{* T} z^{\prime} \cdot d_{0}\right)-\varepsilon \cdot e\right) \cap \operatorname{int}(D)=\emptyset .
$$

Theorem 4.6 (Weak duality) Let $e \in \operatorname{int}(D), \varepsilon \geq 0,(\bar{x}, \bar{y})$ and $\left(x^{\prime}, y^{\prime}, z^{\prime}, y^{*}, z^{*}\right)$ be feasible points for (SOP-I) and (WD), respectively. Suppose that $F$ is D- $\eta$-subinvex at $\left(x^{\prime}, y^{\prime}\right)$ with respect to $\varepsilon \cdot e$ and $G$ is $E$ - $\eta$-invex at $\left(x^{\prime}, z^{\prime}\right)$ with respect to the same $\eta$. Then we have

$$
\bar{y}-y^{\prime}-z^{* T} z^{\prime} \cdot d_{0}+\varepsilon \cdot e \notin-\operatorname{int}(D) .
$$

Proof Assume that

$$
\bar{y}-y^{\prime}-z^{* T} z^{\prime} \cdot d_{0}+\varepsilon \cdot e \in-\operatorname{int}(D) .
$$

Since $G(\bar{x}) \cap(-E) \neq \emptyset$, let $\bar{z} \in G(\bar{x}) \cap(-E), z^{* T} \bar{z} \leq 0$. Hence, we get $z^{* T} \bar{z} \cdot d_{0} \in-D$ and

$$
\bar{y}+z^{* T} \bar{z} \cdot d_{0}-y^{\prime}-z^{* T} z^{\prime} \cdot d_{0}+\varepsilon \cdot e \in-D-\operatorname{int}(D) \subset-\operatorname{int}(D) .
$$

Noticing that $y^{*} \in D^{*} \backslash\left\{0_{Y^{*}}\right\}$ and $y^{* T} d_{0}=1$, we have

$$
y^{* T}\left(\bar{y}-y^{\prime}+\varepsilon \cdot e\right)+z^{* T}\left(\bar{z}-z^{\prime}\right)<0 .
$$

This proves that inequality (4.4) holds. So, the rest of the proof follows from the same arguments as that of the weak duality theorem, Theorem 4.2, for the problem (MWD), we can still get

$$
y^{* T} D F\left(x^{\prime}, y^{\prime}\right)\left(\eta\left(\bar{x}, x^{\prime}\right)\right)+z^{* T} D G\left(x^{\prime}, z^{\prime}\right)\left(\eta\left(\bar{x}, x^{\prime}\right)\right)<0,
$$

which also contradicts the dual constraint of (WD). Thus, $\bar{y}-y^{\prime}-z^{* T} z^{\prime} \cdot d_{0}+\varepsilon \cdot e \notin-\operatorname{int}(D)$, as desired.

Theorem 4.7 (Strong duality) Let $e \in \operatorname{int}(D), \varepsilon \geq 0$, and $(\bar{x}, \bar{y})$ be a weak $\varepsilon \cdot e$-minimizer of (SOP-I). Suppose that, for some $\left(y^{*}, z^{*}\right) \in\left(D^{*} \backslash\left\{0_{Y^{*}}\right\}\right) \times E^{*}$ with $y^{* T} \cdot d_{0}=1$, (3.1) and (3.2) are satisfied for some $\bar{z} \in G(\bar{x}) \cap(-E)$. Then $\left(\bar{x}, \bar{y}, \bar{z}, y^{*}, z^{*}\right)$ is a feasible point for (WD). Furthermore, if the weak duality theorem, Theorem 4.6, between (SOP-I) and (WD) holds, then $\left(\bar{x}, \bar{y}, \bar{z}, y^{*}, z^{*}\right)$ is a weak $\varepsilon \cdot e$-maximizer of (WD).

Proof Since (3.1) and (3.2) are fulfilled, it is obviously that $\left(\bar{x}, \bar{y}, \bar{z}, y^{*}, z^{*}\right)$ is a feasible point for (WD). Next, we show that

$$
\left(K_{2}-\bar{y}-z^{* T} \bar{z} \cdot d_{0}-\varepsilon \cdot e\right) \cap \operatorname{int}(D)=\emptyset .
$$


Let $\left(x^{1}, y^{1}, z^{1}, y_{1}^{*}, z_{1}^{*}\right)$ be a feasible point for (WD) such that $y^{1}+z_{1}^{* T} z_{1} \cdot d_{0} \in K_{2}$ and

$$
y^{1}+z_{1}^{* T} z^{1} \cdot d_{0}-\bar{y}-z^{* T} \bar{z} \cdot d_{0}-\varepsilon \cdot e \in \operatorname{int}(D) .
$$

We find from $z^{* T} \bar{z}=0$ that

$$
y^{1}+z_{1}^{* T} z^{1} \cdot d_{0}-\bar{y}-\varepsilon \cdot e \in \operatorname{int}(D)
$$

This contradicts the weak duality theorem, Theorem 4.6, between (SOP-I) and (WD).

Theorem 4.8 (Converse duality) Let $e \in \operatorname{int}(D), \varepsilon \geq 0$, and $\left(x^{\prime}, y^{\prime}, z^{\prime}, y^{*}, z^{*}\right)$ be a feasible point of the problem (WD) with $z^{\prime} \in G\left(x^{\prime}\right) \cap(-E)$ and $z^{* T} z^{\prime}=0$. Suppose that $F$ is $D-\eta$ subinvex at $\left(x^{\prime}, y^{\prime}\right)$ with respect to $\varepsilon \cdot e$ and $G$ is E- $\eta$-invex at $\left(x^{\prime}, z^{\prime}\right)$ with respect to the same $\eta$. Then $\left(x^{\prime}, y^{\prime}\right)$ is a weak $\varepsilon \cdot e$-minimizer of the problem (SOP-I).

Proof Obviously, $\left(x^{\prime}, y^{\prime}\right)$ is a feasible point of the problem (SOP-I). Let $\left(x^{\prime}, y^{\prime}\right)$ be not a weak $\varepsilon \cdot e$-minimizer of the problem (SOP-I), then

$$
\left(F(\Omega)-y^{\prime}+\varepsilon \cdot e\right) \cap(-\operatorname{int}(D)) \neq \emptyset .
$$

Hence, there exist $\hat{x} \in \Omega$ and $\hat{y} \in F(\hat{x})$ such that

$$
\left(\hat{y}-y^{\prime}+\varepsilon \cdot e\right) \cap(-\operatorname{int}(D)) \neq \emptyset .
$$

Noticing that $y^{*} \in D^{*} \backslash\left\{0_{Y^{*}}\right\}$, we have

$$
y^{* T}\left(\hat{y}-y^{\prime}+\varepsilon \cdot e\right)<0
$$

Again, since $\hat{x} \in \Omega$, we get $G(\hat{x}) \cap(-E) \neq \emptyset$. Let $\hat{z} \in G(\hat{x}) \cap(-E)$, it follows from $z^{*} \in E^{*}$ that $z^{* T} \hat{z} \leq 0$. Noticing that $z^{* T} z^{\prime}=0$, we get

$$
z^{* T}\left(\hat{z}-z^{\prime}\right)=z^{* T} \hat{z} \leq 0
$$

Hence, we obtain

$$
y^{* T}\left(\hat{y}-y^{\prime}+\varepsilon \cdot e\right)+z^{* T}\left(\hat{z}-z^{\prime}\right)<0,
$$

which illustrates that (4.8) is satisfied. By the same arguments as that of the converse duality theorem, Theorem 4.4, for the problem (MWD), we also have

$$
y^{* T} D F\left(x^{\prime}, y^{\prime}\right)\left(\eta\left(\hat{x}, x^{\prime}\right)\right)+z^{* T} D G\left(x^{\prime}, z^{\prime}\right)\left(\eta\left(\hat{x}, x^{\prime}\right)\right)<0 .
$$

This also contradicts the dual constraint of (WD). Hence, $\left(x^{\prime}, y^{\prime}\right)$ is a weak $\varepsilon \cdot e$-minimizer of the problem (SOP-I). 


\section{Vector optimization and variational inequality}

This section is devoted to a discussion of the relationship between approximate solutions of set-valued optimization and that of a kind of vector variational inequality. Let $S$ be a nonempty invex subset of $X$ and $F: X \rightarrow 2^{Y}$ be a set-valued mapping. Considering the following set-valued optimization problem (SOP-II):

$$
\text { (SOP-II) } \begin{cases}\text { minimize } & F(x) \\ \text { subject to } & x \in S .\end{cases}
$$

Let $\bar{x} \in S, \bar{y} \in F(\bar{x})$ and $\eta: X \times X \rightarrow X$ be a map. In the following, it is assumed that $D F(\bar{x}, \bar{y})$ exists, and $\eta(S, \bar{x}):=\{\eta(x, \bar{x}): x \in S\}$ belongs to the domain of $D F(\bar{x}, \bar{y})$.

Now, we consider the vector variational inequality problem (VVIP) $)_{\eta}$, that is, to find $\bar{x} \in S$, $\bar{y} \in F(\bar{x})$ such that

$$
(\mathrm{VVIP})_{\eta} \quad D F(\bar{x}, \bar{y})(\eta(x, \bar{x})) \notin-D, \quad \forall x \in S .
$$

When $\eta(x, \bar{x})=x-\bar{x}$, the vector variational inequality problem (VVIP) was investigated by Liu and Gong [35].

Definition 5.1 (i) The pair $(\bar{x}, \bar{y}) \in \operatorname{graph}(F)$ is called a weak efficient solution of the problem $(\text { VVIP })_{\eta}$, if we have

$$
D F(\bar{x}, \bar{y})(\eta(x, \bar{x})) \notin-\operatorname{int}(D), \quad \forall x \in S
$$

(ii) Let $e \in \operatorname{int}(D)$ and $\varepsilon \geq 0$. The pair $(\bar{x}, \bar{y})$ is called a weak $\varepsilon \cdot e$-efficient solution of the problem (VVIP) $)_{\eta}$, if we have

$$
D F(\bar{x}, \bar{y})(\eta(x, \bar{x}))+\varepsilon \cdot e \notin-\operatorname{int}(D), \quad \forall x \in S .
$$

Theorem 5.2 Let $e \in \operatorname{int}(D)$ and $\varepsilon \geq 0$. If a pair $(\bar{x}, \bar{y})$ is a weak $\varepsilon \cdot e$-minimizer of problem (SOP-II), then $(\bar{x}, \bar{y})$ is a weak $\varepsilon \cdot e$-efficient solution of $(\mathrm{VVIP})_{\eta}$.

Proof Since the pair $(\bar{x}, \bar{y}) \in \operatorname{graph}(F)$ is a weak $\varepsilon \cdot e$-minimizer of (SOP-II), one has

$$
(F(S)-\bar{y}+\varepsilon \cdot e) \cap-\operatorname{int}(D)=\emptyset .
$$

We proceed by contradictions. Assume that there is an $x^{\prime} \in S$ with $y^{\prime}=D F(\bar{x}, \bar{y})\left(\eta\left(x^{\prime}, \bar{x}\right)\right)$ such that

$$
\hat{y}:=y^{\prime}+\varepsilon \cdot e=D F(\bar{x}, \bar{y})\left(\eta\left(x^{\prime}, \bar{x}\right)\right)+\varepsilon \cdot e \in-\operatorname{int}(D) .
$$

Denote $\hat{F}(\cdot):=F(\cdot)+\varepsilon \cdot e$. By the definition of a contingent epiderivative, we get

$$
\left(\eta\left(x^{\prime}, \bar{x}\right), \hat{y}\right) \in \operatorname{epi}(D \hat{F}(\bar{x}, \bar{y}))=T(\operatorname{epi}(\hat{F}),(\bar{x}, \bar{y}))
$$


Then there are a sequence $\left(x_{n}, y_{n}\right)_{n \in \mathbb{N}}$ in $\operatorname{graph}(F)$ with $\left(x_{n}, y_{n}+\varepsilon \cdot e\right)_{n \in \mathbb{N}}$ in epi $(\hat{F})$ and a sequence $\left(\lambda_{n}\right)_{n \in \mathbb{N}}$ of positive real numbers with $(\bar{x}, \bar{y})=\lim _{n \rightarrow \infty}\left(x_{n}, y_{n}+\varepsilon \cdot e\right)$ and

$$
\left(\eta\left(x^{\prime}, \bar{x}\right), \hat{y}\right)=\lim _{n \rightarrow \infty} \lambda_{n}\left(\left(x_{n}, y_{n}+\varepsilon \cdot e\right)-(\bar{x}, \bar{y})\right) .
$$

Hence, we get

$$
\hat{y}=\lim _{n \rightarrow \infty} \lambda_{n}\left(y_{n}+\varepsilon \cdot e-\bar{y}\right) .
$$

Because of the condition (5.2) and (5.3), there is an $n_{0} \in \mathbb{N}$ with

$$
\lambda_{n}\left(y_{n}+\varepsilon \cdot e-\bar{y}\right) \in-\operatorname{int}(D), \quad \forall n \geq n_{0} .
$$

This leads to

$$
y_{n}+\varepsilon \cdot e \in\{\bar{y}\}-\operatorname{int}(D), \quad \forall n \geq n_{0} .
$$

At the same time, since $\left(x_{n}, y_{n}+\varepsilon \cdot e\right) \in \operatorname{epi}(\hat{F})$, there exists $y_{n}^{\prime} \in F\left(x_{n}\right)$ and $d_{n} \in D$ such that $y_{n}+\varepsilon \cdot e=y_{n}^{\prime}+\varepsilon \cdot e+d_{n}$. So, we get from (5.4)

$$
\begin{aligned}
y_{n}^{\prime}+\varepsilon \cdot e & =y_{n}+\varepsilon \cdot e-d_{n} \\
& \in\{\bar{y}\}-\operatorname{int}(D)-D \\
& \subset\{\bar{y}\}-\operatorname{int}(D), \quad \forall n \geq n_{0} .
\end{aligned}
$$

Noticing that $y_{n}^{\prime} \in F\left(x_{n}\right) \subset F(S)$, we get

$$
y_{n}^{\prime}+\varepsilon \cdot e-\{\bar{y}\} \in(F(S)-\{\bar{y}\}+\varepsilon \cdot e) \cap-\operatorname{int}(D) .
$$

This contradicts (5.1). The proof is completed.

For the problem (SOP-II), since every weak minimizer is a weak $\varepsilon \cdot e$-minimizer, we can immediately derive Corollary 5.3.

Corollary 5.3 Let $e \in \operatorname{int}(D)$ and $\varepsilon \geq 0$. If a pair $(\bar{x}, \bar{y})$ is a weak minimizer of problem (SOP-II), then $(\bar{x}, \bar{y})$ is a weak $\varepsilon \cdot$ e-minimizer of $(\mathrm{VVIP})_{\eta}$.

Theorem 5.4 Let $S$ be an invex set with respect to $\eta$ and the set-valued mapping $F: S \rightarrow 2^{Y}$ be $D$ - $\eta$-subinvex on $S$ with respect to $\varepsilon \cdot e$. If $(\bar{x}, \bar{y})$ is a weak efficient solution of $(\mathrm{VVIP})_{\eta}$, then $(\bar{x}, \bar{y})$ is a weak $\varepsilon \cdot e$-minimizer of the problem (SOP-II).

Proof By the assumptions, we get

$$
D F(\bar{x}, \bar{y})(\eta(x, \bar{x})) \notin-\operatorname{int}(D), \quad \forall x \in S .
$$

Assuming that $(\bar{x}, \bar{y})$ is not a weak $\varepsilon \cdot e$-minimizer of (SOP-II), then $\hat{x} \in S, \hat{y} \in F(\hat{x})$ such that

$$
\hat{y}-\bar{y}+\varepsilon \cdot e \in-\operatorname{int}(D) \text {. }
$$


On the other hand, since $F$ is $D-\eta$-subinvex on $S$ with respect to $\varepsilon \cdot e$, we see from Definition 2.7 that there is $\hat{d} \in D$ such

$$
\hat{y}-\bar{y}+\varepsilon \cdot e=D F(\bar{x}, \bar{y})(\eta(\hat{x}, \bar{x}))+\hat{d} .
$$

Thus,

$$
D F(\bar{x}, \bar{y})(\eta(\hat{x}, \bar{x}))=\hat{y}-\bar{y}+\varepsilon \cdot e-\hat{d} \in-\operatorname{int}(D)-D \subset-\operatorname{int}(D),
$$

which contradicts (5.5).

Remark 5.5 Theorem 5.4 generalizes and improves the result of Liu and Gong (see [35], Theorem 7) in the following aspects:

(1) The constraint set which is a convex subset is extended to the invex set.

(2) The objective function, that is, a cone-convex set-valued mapping, is extended to cone-subinvex.

Remark 5.6 This note only presents the relationships between a kind of generalized variational-like inequalities and set-valued optimization problem. However, we do not discuss the relationships of other kinds of variational-like inequalities and set-valued optimization, and the existence problems of variational-like inequalities are not involved. For more details related to these problems, we refer the reader to $[27,28,36]$.

\section{Conclusions and remarks}

In this paper, we focus on the approximate solutions in set-valued optimization. We present the notion of cone-subinvex set-valued maps and investigate its properties. A sufficient optimality condition and two types dual theorems are established for weakly approximate minimizers under the assumption of cone-subinvexity. We also discuss the relationships between a kind of vector variational inequality and set-valued optimization. Under the assumption cone-subinvexity, it shows that the weakly approximate minimizers of set-valued optimization are characterized by the weakly approximate solution of a kind of vector variational inequality.

It is worthy underlining that Ansari and Jahn [36] defined the $\mathbb{T}$-epiderivative of a setvalued map, which includes the contingent epiderivative as its special case. They provided necessary and sufficient conditions for a solution of a set-valued problems and some existence results for solutions of set-valued optimization problems and a generalized vector $\mathbb{T}$-inequality problem under the assumption of cone-convexity for set-valued maps. It is possible to extend the notion of cone-subinvexity in the setting of $\mathbb{T}$-epiderivative and to deal with similar problems for approximate solutions in set-valued optimization, such as optimality conditions and duality. This must be an interesting and meaningful work. 


\section{Acknowledgements}

This research was supported by Natural Science Foundation of China under Grant No. 11361001; Ministry of Education Science and technology key projects under Grant No. 212204; Natural Science Foundation of Ningxia under Grant No. NZ14101. The authors are grateful to the anonymous referees who have contributed to improve the quality of the paper.

Received: 10 June 2015 Accepted: 23 September 2015 Published online: 09 October 2015

\section{References}

1. Aubin, JP, Frankowsa, H: Set-Valued Analysis. Birkhäuser, Boston (1990)

2. Luc, DT: Theory of Vector Optimization. Springer, Berlin (1989)

3. Jahn, J: Vector Optimization: Theory, Applications and Extensions. Springer, Berlin (2003)

4. Chen, GY, Huang, XX, Yang, XQ: Vector Optimization: Set-Valued and Variational Analysis. Springer, Berlin (2005)

5. Jahn, J, Rauh, R: Contingent epiderivatives and set-valued optimization. Math. Methods Oper. Res. 46, 193-211 (1997)

6. Jahn, J, Rauh, R: The existence of contingent epiderivatives for set-valued maps. Appl. Math. Lett. 16(8), 1179-1185 (2003)

7. Cheng, GY, Jahn, J: Optimality conditions for set-valued optimization problem. Math. Methods Oper. Res. 48, 187-200 (1998)

8. Rodríquez-Marín, L, Sama, M: About contingent epiderivatives. J. Math. Anal. Appl. 327(2), 754-762 (2007)

9. Rodríguez-Marín, L, Sama, M: Variational characterization of the contingent epiderivative. J. Math. Anal. Appl. 335(2), 1374-1382 (2007)

10. Gong, $\mathrm{XH}$ : Optimality conditions for Henig and globally proper efficient solutions with ordering cone has empty interior. J. Math. Anal. Appl. 307, 12-31 (2005)

11. Jiménez, B, Novo, V, Sama, M: Scalarization and optimality conditions for strict minimizers in multiobjective optimization via contingent epiderivatives. J. Math. Anal. Appl. 352(2), 788-798 (2009)

12. Yu, GL: Optimality of global proper efficiency for cone-arcwise connected set-valued optimization using contingent epiderivative. Asia-Pac. J. Oper. Res. 30(3), 1340004 (2013)

13. Hanson, MA: On sufficiency of Kuhn-Tucker conditions. J. Math. Anal. Appl. 80, 545-550 (1981)

14. Luc, DT, Malivert, C: Invex optimization problems. Bull. Aust. Math. Soc. 49, 47-66 (1992)

15. Sach, PH, Craven, BD: Invex multifunction and duality. Numer. Funct. Anal. Optim. 12, 575-591 (1991)

16. Lalitha, CS, Dutta, J, Govll, MG: Optimality criteria in set-valued optimization. J. Aust. Math. Soc. 75, 221-231 (2003)

17. Alonso, M, Rodríguez-Marín, L: Optimality conditions for a nonconvex set-valued optimization problem. Comput. Math. Appl. 56, 82-89 (2008)

18. Oveisiha, M, Zafarani, J: On characterization of solution sets of set-valued pseudoinvex optimization problems. J. Optim. Theory Appl. 163(2), 387-398 (2014)

19. Das, K, Nahak, C: Sufficient optimality conditions and duality theorems for set-valued optimization problem under generalized cone convexity. Rend. Circ. Mat. Palermo 63, 329-345 (2014)

20. Davinder, B: Lagrangian duality for preinvex set-valued functions. J. Math. Anal. Appl. 214, 599-612 (1997)

21. Long, XJ, Peng, JW: Lagrangian duality for vector optimization problems with set-valued mappings. Taiwan. J. Math 17(1), 287-297 (2013)

22. Azimov, AY: Duality for set-valued multiobjective optimization problems, part 1: mathematical programming J. Optim. Theory Appl. 137, 61-74 (2008)

23. Heydea, F, Schrage, C: Continuity concepts for set-valued functions and a fundamental duality formula for set-valued optimization. J. Math. Anal. Appl. 397, 772-784 (2013)

24. Sach, PH, Lee, GM, Kim, DS: Strong duality for proper efficiency in vector optimization. J. Optim. Theory Appl. 130, 139-151 (2006)

25. Sach, $\mathrm{PH}$, Tuan, LA: Strong duality with proper efficiency in multiobjective optimization involving nonconvex set-valued maps. Numer. Funct. Anal. Optim. 30, 371-392 (2009)

26. Giannessi, F: Variational Inequalities and Complementarity Problems. Wiley, New York (1980)

27. Al-Homidan, S, Ansari, QH: Relations between generalized vector variational-like inequalities and vector optimization problems. Taiwan. J. Math. 16(3), 987-998 (2012)

28. Ansari, QH, Rezaei, M, Zafarani, J: Generalized vector variational-like inequalities and vector optimization. J. Glob. Optim. 53(2), 271-284 (2012)

29. Jayswal, A, Choudhury, S, Verma, RU: Exponential type vector variational-like inequalities and vector optimization problems with exponential type invexities. J. Appl. Math. Comput. 45, 87-97 (2014)

30. Jayswal, A, Choudhury, S: Exponential type vector variational-like inequalities and nonsmooth vector optimization problems. J. Appl. Math. Comput. (2014). doi:10.1007/s12190-014-0828-8

31. Alonso-Durán, M, Rodríguez-Marín, L: On approximate solutions in set-valued optimization problems. J. Comput. Appl. Math. 236, 4421-4427 (2012)

32. Gao, Y, Hou, SH, Yang, XM: Existence and optimality conditions for approximate solutions to vector optimization problems. J. Optim. Theory Appl. 152, 97-120 (2012)

33. Long, XJ, Li, XB, Zeng, J: Lagrangian conditions for approximate solutions on nonconvex set-valued optimization problems. Optim. Lett. 7, 1847-1856 (2013)

34. Zhou, ZA, Yang, XM, Peng, JW: $\varepsilon$-Optimality conditions of vector optimization problems with set-valued maps based on the algebraic interior in real linear spaces. Optim. Lett. 8, 1047-1061 (2014)

35. Liu, W, Gong, XH: Proper efficiency for set-valued vector optimization problems and vector variational inequalities. Math. Methods Oper. Res. 51, 443-457 (2000)

36. Ansari, QH, Jahn, J: $\mathbb{T}$-Epiderivative of set-valued maps and its application to set optimization and generalized variational inequalities. Taiwan. J. Math. 14(6), 2447-2468 (2010) 